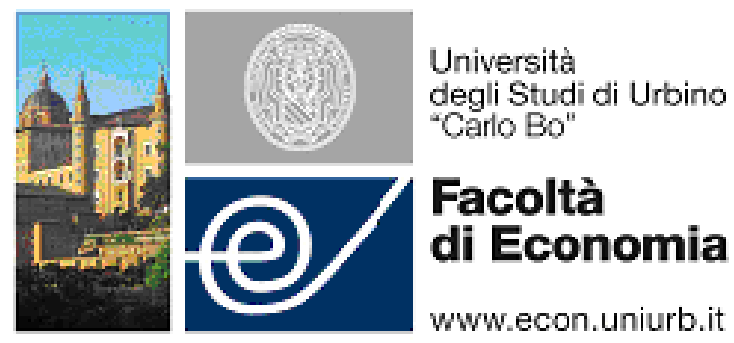

WP-EMS

Working Papers Series in Ec onomics, Mathematics and Statistics

\title{
"The dynamics of the NAIRU model with two switching regimes"
}

- Fabio Tramontana, (U. Ancona)

- Laura Gardini, (U. Urbino)

- Piero Ferri, (U. Bergamo) 


\title{
The dynamics of the NAIRU model with two switching regimes
}

\author{
${ }^{1}$ Fabio Tramontana, ${ }^{2}$ Laura Gardini, ${ }^{3}$ Piero Ferri \\ ${ }^{1}$ Department of Economics, University of Ancona, Italy \\ ${ }^{2}$ Department of Economics and Quantitative Methods, University of \\ Urbino, Italy \\ ${ }^{3}$ Department of Economics, University of Bergamo, Italy
}

\begin{abstract}
We consider a model of inflation and unemployment proposed in Ferri et al. (JEBO, 2001), in which the dynamics are described by a discontinuous piecewise linear map, made up of two branches. We shall show that the bounded dynamics may be classified in two cases: we may have either regular dynamics with stable cycles of any period or quasiperiodic trajectories, or only chaotic dynamics (pure chaos in which a unique absolutely continuous invariant ergodic measure exists, and structurally stable), in a rich variety of cyclical chaotic intervals. The main results are the analytical formulation of the border collision bifurcation curves, through which we give a complete picture of the possible outcomes of the model.
\end{abstract}

JEL classification: E31, E32, C62.

Keywords: Phillips curve; Regime switching; NAIRU; Nonlinearities; Discontinuous maps.

\section{Introduction}

The recent events that characterize the world economy are challenging for economics that has evolved under the double pressure of internal forces and external facts. From an empirical point of view, the stylized facts characterizing the economy are changing dramatically. The question "is the business cycle obsolete?" seems to have been replaced by an investigation of the forces that are transforming the actual recession into a likely slump. In this perspective, two are the methodological tenets to be taken into consideration. First of all, the emphasis must be put on endogenous dynamics, as Hicks [21] and Minsky [35] stressed long time ago (see also [18]). Secondly, the focus should be put on a medium-run 
horizon, where both short-run and long-run vibrations do not occupy the center stage.

In the present paper we take into considerations these challenges by focusing on the dynamics of inflation and unemployment, which are at the core of macroeconomic analysis. These themes have been traditionally studied by the literature on the Phillips curve based upon the so-called NAIRU (the non accelerating-inflation rate of unemployment ([27]). The NAIRU has been criticized from different point of views (see [2] and [5]). However the criticism to its attraction properties has been less compelling ([17]). This is the central theme of the present work that considers a model of inflation and unemployment proposed in [16], where these phenomena are studied with two innovations. On one hand, the Phillips curve interacts with aggregate demand problems so that both unemployment and inflation are studied within a system perspective. On the other, it is assumed that a regime switching affects the parameters of the model in a way that the dynamics of unemployment at time $t, u_{t}$, and consequently of inflation, are described by a one-dimensional discontinuous map consisting of two linear branches, given by (as we shall describe in Section 2):

$$
u_{t+1}=\theta\left(u_{t}\right)= \begin{cases}\theta_{L}\left(u_{t}\right)=\omega u_{t}+(1-\omega) u^{*} & \text { if } \quad u_{t} \leq u^{S} \\ \theta_{R}\left(u_{t}\right)=\omega^{\prime} u_{t}+\left(1-\omega^{\prime}\right) u^{* \prime} & \text { if } \quad u_{t}>u^{S}\end{cases}
$$

where $u^{S}$ is the threshold level of unemployment at which a regime switching happens, $u^{*}$ and $u^{* \prime}$ are the NAIRU for regimes $\mathrm{L}$ and $\mathrm{R}$, respectively. That is, when the level of unemployment exceeds $u^{S}$ a change of strategy occurs, so that the "Left side" with respect to $u^{S}$ represents the regime of low unemployment, or regime L, while the "Right side" with respect to $u^{S}$ represents the regime of high unemployment, or regime R. The two slopes are respectively $\omega$ and $\omega^{\prime}$.

Similarly, a two-phase system exists for the rate of inflation:

$$
\pi_{t+1}=\Theta\left(\pi_{t}\right)=\left\{\begin{array}{lll}
\Theta_{L}\left(\pi_{t}\right)=\omega \pi_{t}+(1-\omega) \pi^{*} & \text { if } \pi_{t} \leq \pi^{S} \\
\Theta_{R}\left(\pi_{t}\right)=\omega^{\prime} \pi_{t}+\left(1-\omega^{\prime}\right) \pi^{* \prime} & \text { if } \quad \pi_{t}>\pi^{S}
\end{array}\right.
$$

where $\pi^{S}=m+u^{S} / \alpha$ is the threshold for the inflation rate ${ }^{1}$. Clearly regime $\mathrm{L}$ is the virtuous one, with low unemployment and low rate of inflation, while regime $\mathrm{R}$ is the vicious one, characterized by high unemployment and high rate of inflation. And the dynamic behavior of the two variables $u_{t}$ and $\pi_{t}$ is qualitatively the same, due to the linear relation between them, $\pi_{t}=m+\frac{u_{t}}{\alpha}$, as recalled in Section 2 .

\footnotetext{
${ }^{1}$ as described in Section 2, $m$ is the rate of growth of money and the $\alpha$ measures the impact of inflation on the aggregate demand.
} 
The results are very encouraging. First of all, inflation and deflation can be explained by the same set of forces which can produce both low and high rates of unemployment. Secondly, the model can generate very complex dynamics which are compatible with the coexistence of accelerations and decelerations, without generating runaway situations. Finally, policy implications are less straightforward than those originating from the traditional approach. In fact, the NAIRU is not necessarily a single value, nor necessarily reachable and therefore it is very difficult to measure. What happens is that an interval of values for unemployment (also stressed by Solow in [48]) becomes compatible with a bounded situation of inflation dynamics.

The dynamic behavior in our model (1) strongly depends on the values of the two slopes $\omega$ and $\omega^{\prime}$ of the curves $\theta_{L}\left(u_{t}\right)$ and $\theta_{R}\left(u_{t}\right)$ respectively. Following Ferri et al. [16], we shall consider the dynamic in three different cases.

(I): In the first case the two slopes $\omega$ and $\omega^{\prime}$ are both less than 1. This case is characterized by regular dynamics. In [16] the particular case with $0<\omega^{\prime}<\omega<1$ was considered ${ }^{2}$, showing that cycles of any period may exist. Here we shall completely characterize the occurring dynamics. We shall see that cycles may exist not only with any period, but also with a different number of points in the two regimes $\mathrm{L}$ and $\mathrm{R}$. That is: suitable values of $\omega$ and $\omega^{\prime}$ exist such that the dynamics are convergent to a periodic orbit of period $k>1$ with several combinations of $p$ points in the regime $\mathrm{L}$ and $(k-p)$ points in the regime $\mathrm{R}$. For each of such cycles a suitable parameter region is shown to exist, and each existing cycle is always globally asymptotically stable. When a stable cycle does not exist the dynamics are quasiperiodic with trajectories dense on an interval.

(II): In the second case the two slopes $\omega$ and $\omega^{\prime}$ are both larger than 1. This is clearly a case with true chaotic dynamics (as already shown in [16]), and we shall show that chaos may occur in cyclical intervals (not only in one unique interval).

(III): In the third case the two slopes $\omega$ and $\omega^{\prime}$ are one lower than 1 and the other larger than 1 . This case was mentioned in [16], but not investigated. As we shall see, this is the case with a rich variety of dynamics, which however may be summarized as in the two previous cases. In fact, there may be either regular dynamics with bounded quasiperiodic trajectories or asymptotically stable cycles of any period $k$, or a chaotic dynamics (true robust chaos) in $k$-cyclical chaotic intervals of any period $k \geq 1$. As we shall see, there will be a sudden transition,

\footnotetext{
${ }^{2}$ representing the case in which the slope of the Phillips curve in regime $(\mathrm{L})$ is larger than in regime $(\mathrm{R})$.
} 
leading from the regular region in which the dynamics are the same as those occurring in case (I), to the chaotic one, in which the dynamics are the same as those occurring in case (II).

The goal of the present paper is to describe the bifurcation curves which can be analytically determined as a function of the parameters, and a detailed description of the dynamics in the three cases described above.

The study of the bifurcations in a piecewise-smooth system is quite different from those occurring in the smooth one. It is nowadays well known that in smooth systems the dynamics may evolve from a regular dynamic behavior to a complex one via a sequence of bifurcations (as, for example, routes to chaos via Feigenbaum cascades of period doubling bifurcations). While in piecewise-smooth systems Border-Collision Bifurcations (BCB for short) may occur. And in piecewise-linear systems (which is the case in this paper) mainly $\mathrm{BCB}$ and contact bifurcations ${ }^{3}$ occur (the local bifurcations, when occurring, are degenerate). It is classified as border-collision any contact between an invariant set of a map with the border of its region of definition, and this may, or may not, produce a bifurcation. The term border-collision bifurcation was used for the first time by Nusse and Yorke in 1992 [41], (see also [42]) and it is now widely used in this context (i.e. for piecewise smooth maps), although the study and description of such border collision bifurcations started several decades before that papers. These bifurcations have been widely studied in the recent years, mainly because of their relevant applications in Engineering ([14], [6], [3], [4]). Also before and after the works by Nusse and Yorke the bifurcations associated with piecewise smooth maps were studied in some papers, even if the bifurcations were not called of border-collision, see for example [36], [37], [30], [31], [32] and others. In particular, in [14] some results by Feigen are republished, which were already printed in $1978^{4}$ (but unknown to a wide public). We may also go further back, citing the works by Leonov in the 60th, [28], [29]. In his works, Leonov described several bifurcations, giving a recurrence relation to find the analytic expression of the family of bifurcations occurring in a one-dimensional piecewise linear map with one discontinuity point, which also is still mainly unknown. Some of his results will be also recalled later, because they are our starting point,

\footnotetext{
${ }^{3}$ We recall that following [38] a contact bifurcation occurs when two invariant sets of different nature have a contact in one or more points. The dynamic effect of a contact bifurcation may be of several different kinds, and depends on the nature of the invariant sets and on the map.

${ }^{4}$ It is worth noticing that the clear and simple analysis performed by Feigen in 1978 is the first one for $n$-dimensional piecewise linear continuous maps, with $n>1$.
} 
in order to understand and describe the bifurcations occurring in our model.

However, it is particularly in the Economic context that piecewise smooth models may arise, and in fact economic models described by onedimensional piecewise smooth or discontinuous maps are already present in the literature. A pioneer in this context is Richard Day. In fact, his first studies of piecewise smooth models start more then 25 years ago, and his results, found independently on the authors cited above, on the bifurcation curves in such models are still extremely up to date (although they were not called border-collision bifurcation curves). We mention, in particular, Day's results in [10], [11], [12], [13], which have been used also recently in [34]. Clearly other economists were interested in the bifurcations of piecewise-smooth system, and it is worth of mention also the works by Cars Hommes ([22], [23], [24], [25]). The BCBs have been considered also recently in several economic models described by continuous functions, for example in Business Cycles models in [45], [46], [49]; in Growth models in [50], [51], [33], [39], [40], [19]. While BCBs in discontinuous models also occur in Duopoly models in [43], [44], [52].

Recently, a one-dimensional piecewise-linear discontinuous map was considered in the one-sector growth model proposed by Böhm and Kaas in $[7]^{5}$, which is similar to our model. The case in which these authors were interested in corresponds to the one called above of regular dynamics. In [7] the authors prove that all the existing cycles are globally attracting, and refer to a remarkable paper by Keener [26] to state that for a set of parameter values of zero Lebesque measure the attracting set is a Cantor set, which however they have never observed. Indeed it is correct, because the attracting set in regular dynamics cannot be a Cantor set. As we shall recall in Sections 3 and 5 in such a case the asymptotic dynamics are either periodic or quasiperiodic (and dense in an interval). In the paper by Keener [26] there is an important results: it is shown that for increasing piecewise smooth discontinuous maps (not only linear), there exists a sharp transition from regular dynamics (no chaotic set can exist) to the chaotic one (no stable cycle can exist). This is also what occurs in our model (as we shall prove in Section 5). Moreover, in [7] the authors remark a surprisingly rich occurrence of periods in the cycles, and the same occurs in our model: the rich structure of periodicity regions with cycles of any period and with periodic points in several positions between the $\mathrm{L}$ and $\mathrm{R}$ regions will be here fully described.

The plan of the work is as follows. In Section 2 we shall describe the model. In Section 3 we shall study the dynamics occurring in case (I): the

\footnotetext{
${ }^{5}$ We thank Cars Hommes for providing this reference.
} 
analytical bifurcation curves associated with the BCBs of the maximal cycles will be given, as well as the mechanism to find all the existing ones. In Section 4 we shall characterize the divergent case (II), showing which kind of contact bifurcations occur, bounding in the parameter space the region of existence of robust chaos (separating the case of divergence almost everywhere). In Section (5) we shall consider the intermediate case (III), determining (in agreement with the result in [26]) the exact value at which a drastic change occurs: from regular dynamics (only attracting cycles can exist characterized by the same BCB curves determined in Section 3, or quasiperiodic trajectories) to chaotic dynamics (robust chaos in cyclical intervals). Some conclusions are given in Section 6.

\section{The model}

In the literature, the dynamics of inflation and unemployment is generally described by the Phillips curve. In the so-called accelerationist version it can be written in the following way:

$$
\pi_{t}=\pi_{t-1}-\tau\left(u_{t}-u^{*}\right)
$$

where $\pi_{t}$ is the inflation rate at time $t, u_{t}$ the rate of unemployment and $u^{*}$ the non-accelerating inflation rate of unemployment, or NAIRU. The positive parameter $\tau$ measures the impact of the labor market conditions on the inflation dynamics. Both the specification and the model itself have been criticized in the literature. For instance, according to Akerlof et al. [2] (p. 2) "...the inadequacy of such models has been demonstrated forcefully in recent years, as low and stable rates of inflation coexisted with a wide range of unemployment rates. If there were a single, relatively constant natural rate, one should have seen inflation slowing significantly when unemployment was above the rate, and rising when it was below". To remedy this inadequacy, they propose a nonlinear equation. Ball and Mankiw [5] (p. 115), on the contrary, state that "the NAIRU is a useful piece of business cycle theory... Once this short-run trade-off is admitted, there must be some level of unemployment consistent with stable inflation". These authors practically admit that there are difficulties in estimating the concept for the simple reason that it can change over time. Their proposal is to assume a changing NAIRU. The present paper takes into account these suggestions and develops them in a particular way, by referring to a regime switching technique.

Before dealing with these problems, one must close the model. In fact, while the Phillips curve represents the supply side of the economy, 
an aggregate demand (AD) must be added:

$$
\pi_{t}=m+\frac{u_{t}}{\alpha}
$$

where $m$ is the (constant) rate of growth of money and $\alpha$ is a parameter that depends on the specification of the model. In fact, the demand equation is traditionally formulated in terms of rate of growth of income. In order to use the rate of unemployment, one has to refer to the Okun's law that states a negative relationship between the two variables. $\alpha$ belongs therefore to this technological universe, even though its meaning can change in a different specification of the model, as will be shortly shown. Combining (3) and (4) we obtain the dynamics of unemployment:

$$
u_{t+1}=\omega u_{t}+(1-\omega) u^{*}
$$

and the dynamics of inflation:

$$
\pi_{t+1}=\omega \pi_{t}+(1-\omega) \pi^{*}
$$

where $\omega=1 /(1+\tau \alpha)$ and $\pi^{*}=m+u^{*} / \alpha$.

Equations (5) and (6) are both linear and the value of $\omega$ determines the stability of the NAIRU. For $\omega<1$ the NAIRU is asymptotically stable whereas it becomes unstable (divergent) for $\omega>1$.

More interesting dynamics emerge introducing nonlinearity in the equations governing the evolution of inflation rate and unemployment. Following [16] we adopt a regime switching to introduce the nonlinearity in the model. In particular, we consider the case in which the regime switching concerns the parameters of both the unemployment and the inflation equations, giving rise to the maps (1) and (2).

Let us justify the changes in the parameter $\omega$ from an economic point of view. A component of $\omega$ is $\tau$, and $1 / \tau$ represents the sacrifice ratio implicit in the Phillips curve. As stressed by Sbordone [47] (p. 3) "A flatter Phillips curve carries the implication that, for a given degree of inflation persistence, reducing inflation involves a higher 'sacrifice ratio' than otherwise, namely it requires enduring a longer period of unemployment above the natural rate for every percentage point of reduction in inflation. On the other hand,...a flatter Phillips curve implies that an overheated economy will tend to generate a smaller increase in inflation". There are econometrics studies quoted in the above article showing that the sacrifice ratio change in time.

The nature of the parameter $\tau$ depends on the specification of the model. For instance, if the above equations are expressed in terms of forward expectations, they usually do not include those terms that Layard et al. [27] define as nominal rigidities parameters. In this case, the 
Phillips curve becomes a New-Keynesian Phillips curve (see [53]) and changes in $\tau$ can be attributed to changes either in the labor market or in the product market. One can assume that the real rigidity parameter can vary according to the state of the labor market, indexed by $u^{S}$. Layard et al. [27] offers many reasons why the bargaining power of workers can change according to the state of the labor market.

Mutatis mutandis, $\tau$ can change because the conditions on the product market vary. For instance, some studies (see [8]) have linked variations in the slope of the Phillips curve to globalization. According to them, both a decline in the autoregressive coefficient and a fall in the slope have been experienced in recent periods. Some observations are to be raised at this stage of the analysis in order to understand if they support our hypotheses. First of all, these changes must be reversible in order to be considered within a regime switching framework. In the case of globalization, this implies that its intensity can reverse in time and that the unemployment threshold can be a relevant barrier. Finally, the changes in the autoregressive parameters, measuring persistence, imply that some nominal inertia is present. Some authors have estimated in other contexts (see [9]), that there is a nonlinear relationship between these parameters and the level of inflation. Since this is related to the level of unemployment, the findings are in keeping with our hypothesis. When inflation is high, people become more concerned with inertia.

Since $\tau$ is positive, the case $\omega<1$ occurs when $\alpha$ is a positive constant. Thus, if one wants to deal with the case $\omega>1$ the only possibility is to have $\alpha$ negative. In the canonical dynamic $\mathrm{AD}$ equation, the velocity of money is assumed to be constant. In that case $\alpha$ is derived from the Okun's law linking growth to unemployment. In the case of financial turbulence, however, velocity can change and this can have an impact on $\alpha$. In fact, the velocity of circulation can be a function of inflation, growth and the degree of uncertainty (see [15], [53]). In this case $\alpha$ ceases to reflect only technological aspect to be influenced also by financial factors. In periods of liquidity trap stressed by Keynes or of debt deflation underlined by Minsky ([35]) there can be a negative relationship between money growth and price dynamics due to the offsetting pattern of the velocity of circulation. ${ }^{6}$

In the following sections we focus only on the unemployment map (1)

\footnotetext{
${ }^{6}$ Another possibility consists in referring to a medium-run model and suppose that the relationship between the rate of growth of income and $u$ is more complex than that established by Okun. According to [1], this relationship is nonlinear and therefore can justify different values of $\alpha$.
} 
knowing that when the low (resp. high) unemployment regime is active than the low (resp. high) inflation rate regime is active too.

We notice that our model (1) ultimately depends on 5 parameters: the two slopes $\omega$ and $\omega^{\prime}$, the two NAIRU constants influencing the width of the jump in the discontinuity point: $u^{*}$ and $u^{* \prime}$, and the position of the discontinuity point: $u^{S}$. By rescaling the state variable $u_{t}$ we could set one of the parameters to 1 , leaving only 4 parameters which influences the dynamic behaviors. However, due to the economic interpretation of the model, we keep all the parameters as they are shown in (1) and the bifurcation curves will be expressed analytically as a function of all the five parameters, so as to see immediately where they play a role (and setting one of them equal to one is something which can be done whenever needed).

The fixed points of (1) may exist or not. Let us denote by $P^{*}$ the fixed point belonging to the $\mathrm{L}$ region, given by $P^{*}=u^{*}$, which exists when $u^{*} \leq u^{S}$, and its stability depends on the value of the slope $\omega$. A second possible fixed point, belonging to the $\mathrm{R}$ region, given by $Q^{*}=u^{* \prime}$, exists when $u^{* \prime} \geq u^{S}$, and its stability depends on the value of the slope $\omega^{\prime}$.

\section{Analysis of case (I) $\omega<1, \omega^{\prime}<1$}

In this section we are going to describe the properties of our model in (1) when the two positive slopes $\omega$ and $\omega^{\prime}$ are both less than 1 . This case is characterized by regular dynamics. Indeed, whenever a $k$-cycle can exist, with $p$ periodic points in the $\mathrm{L}$ region and $(k-p)$ in the $\mathrm{R}$ one, then the related eigenvalue is given by

$$
\lambda=\omega^{p} \omega^{\prime(k-p)}
$$

which is positive and less then 1 , denoting the local stability of the cycle. In particular, if the fixed point $P^{*}=u^{*}$ belonging to the $\mathrm{L}$ region exists, then it attracts all the points in the $\mathrm{L}$ region (i.e. for $u \leq u^{S}$ ). If the fixed point $Q^{*}=u^{* \prime}$ belonging to the $\mathrm{R}$ region exists, then it attracts all the points in the $\mathrm{R}$ region (i.e. for $u>u^{S}$ ). When only one equilibrium point exists then it is globally attracting. When both exist then those described above (the $\mathrm{L} / \mathrm{R}$ regions) are their basins of attraction, and we notice that the values of the jump in the discontinuity point are such that $u^{m}=\theta_{R}\left(u^{S}\right)$ tends to $P^{*}$ while $u^{M}=\theta_{L}\left(u^{S}\right)$ tends to $Q^{*}$. It follows that when the parameters satisfy

$$
u^{* \prime}<u^{S}<u^{*}
$$

then no fixed point exists. Indeed this is the regime which allows for stable cycles of any period, and all belong to the so-called absorbing 
interval made up of the maximum $u^{M}$ taken from the $\mathrm{L}$ region and the minimum $u^{m}$ taken from the $\mathrm{R}$ region:

$$
I=\left[u^{m}, u^{M}\right]
$$

where

$$
\begin{aligned}
& u^{m}=\theta_{R}\left(u^{S}\right)=\left(1-\omega^{\prime}\right) u^{* \prime}+\omega^{\prime} u^{S}<u^{S} \\
& u^{M}=\theta_{L}\left(u^{S}\right)=(1-\omega) u^{*}+\omega u^{S}>u^{S}
\end{aligned}
$$

Fig.1 gives an example of the shape of the map in this case (I).

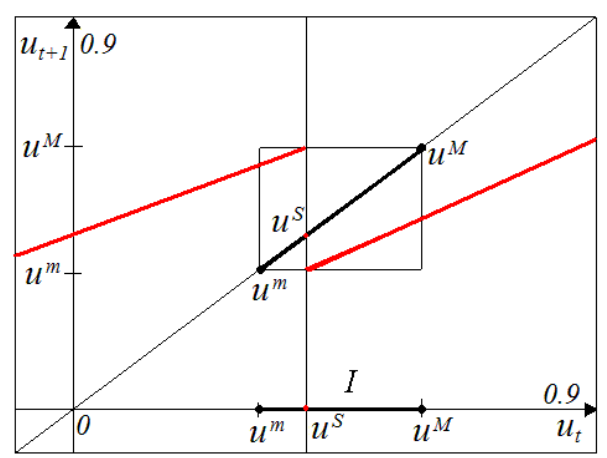

Fig.1. Shape of the map $\theta\left(u_{t}\right)$ at $u^{* \prime}=0.2, u^{S}=0.4, u^{*}=0.8, \omega=0.5, \omega^{\prime}=0.6$.

That $I$ is an absorbing interval comes from the fact that any initial condition $u<u^{m}$ has an increasing trajectory entering $I$ in a finite number of steps, and similarly any initial condition $u>u^{M}$ has a decreasing trajectory entering $I$ in a finite number of steps. On the other hand $I$ is invariant $(I=\theta(I))$ which means that a point in $I$ has a trajectory which cannot escape $I$, so that its omega limit set must be a set inside the interval itself.

Let us now describe how several kinds of cycles can exist. As we shall see, the region of existence of a $k$-cycle in the parameter space is bounded by two BCB curves. We describe first those related with the so called maximal cycles (also called principal cycles or cycles of the first degree of complexity), which are the regions of existence of a $k$-cycle (for any integer $k>1$ ) having one point in the region $\mathrm{R}$ and $(k-1)$ points in the other region L. As a first example let us consider the region associated with a 4 -cycle. When such a cycle exists, we must have a fixed point of the $\operatorname{map}^{7} \theta_{L} \circ \theta_{L} \circ \theta_{L} \circ \theta_{R}$ (as well as for the other compositions of maps obtained cyclically from this one), that is, by denoting the periodic points of the cycle as $\left\{u_{i}\right\}$ with $u_{1}>u^{S}$ and the

\footnotetext{
${ }^{7}$ the symbol $\circ$ represents the composition of functions, that is $g \circ f()=.g(f()$.$) .$
} 
other points $u_{2}<u_{3}<u_{4}<u^{S}$, we have $\theta_{L}^{3} \circ \theta_{R}\left(u_{1}\right)=u_{1}$. Such a cycle disappears by border collision bifurcation either when the periodic point on the right $u_{1}$ is at its lowest value: the discontinuity point $u_{1}=u^{S}$, or when it reaches the rightmost value, $u_{1}=u^{M}$, which also means that the periodic point which is its preimage has reached the discontinuity point: $u_{4}=u^{S}$. In our case the two situations are shown in Fig.2a and Fig.2b, respectively.

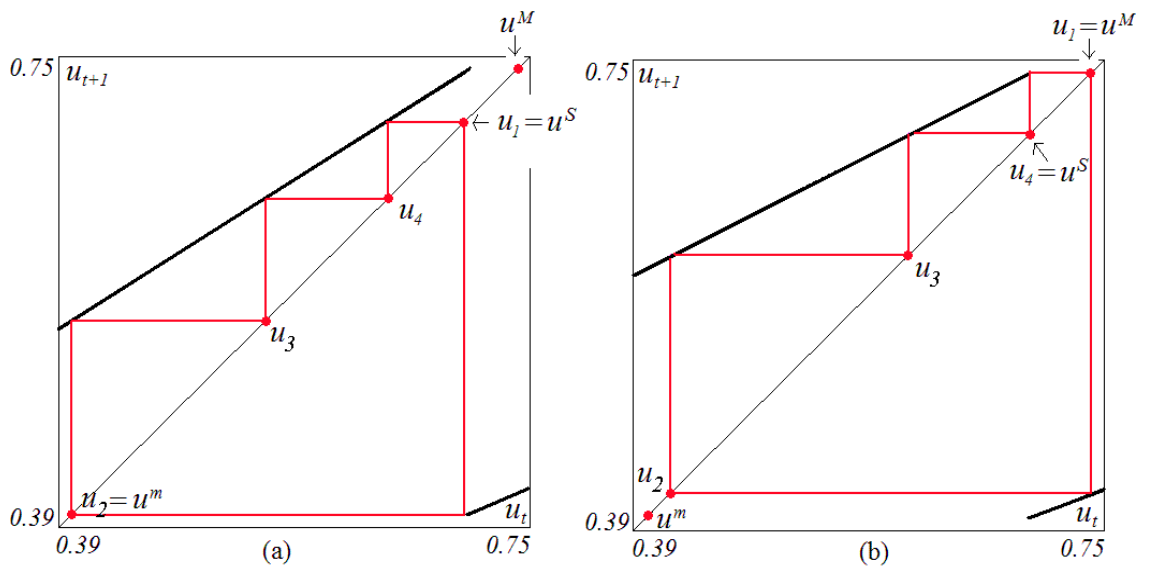

Fig. $2 u^{*}=0.8, u^{* \prime}=0.2, u^{S}=0.7$ and $\omega^{\prime}=0.4$. In (a): $\omega=0.63$; in (b): $\omega=0.51$.

Similarly we can reason with a maximal cycle of any period $k$, and the conditions given above are used to find the conditions for the two BCBs associated with a maximal $k$-cycle with periodic points $\left\{u_{i}\right\}$ having $u_{1}>u^{S}$ and the other points $u_{2}<\ldots<u_{k}<u^{S}$. In formulae we get:

$$
\begin{aligned}
u_{2}= & \omega^{\prime} u_{1}+\left(1-\omega^{\prime}\right) u^{* \prime} \\
u_{3}= & \omega u_{2}+(1-\omega) u^{*}=\omega \omega^{\prime} u_{1}+\omega\left(1-\omega^{\prime}\right) u^{* \prime}+(1-\omega) u^{*} \\
& \cdots \\
u_{k+1}= & \omega^{k-1} \omega^{\prime} u_{1}+\omega^{k-1}\left(1-\omega^{\prime}\right) u^{* \prime}+\left(1-\omega^{k-1}\right) u^{*}
\end{aligned}
$$

and a $k$-cycle occurs when the equality $u_{1}=u_{k+1}$ holds, which gives:

$$
u_{1}=\frac{u^{*}-\omega^{k-1}\left(u^{*}+\omega^{\prime} u^{* \prime}-u^{* \prime}\right)}{\left(1-\omega^{k-1} \omega^{\prime}\right)}
$$

Thus we have the analytical expression of the periodic points of such $k$-cycles: one point, $u_{1}$, comes from (10), and the others are easily found by applying the map $\theta_{L}$. In particular we have:

$$
u_{k}=\frac{u^{*}+\omega^{k-2}\left[u^{* \prime} *\left(1-\omega^{\prime}\right)+\omega^{\prime} u^{*}(1-\omega)-u^{*}\right]}{\left(1-\omega^{k-1} \omega^{\prime}\right)}
$$


Moreover, as commented above, from these expressions we can also obtain the conditions on the parameters at which the two BCBs occur. In fact, the equation of one BCB curve is obtained when $u_{1}=u^{S}$, which gives, from (10):

$$
B C B_{k}^{1}: u^{S}\left(1-\omega^{k-1} \omega^{\prime}\right)-u^{*}+\omega^{k-1}\left(u^{*}+\omega^{\prime} u^{* \prime}-u^{* \prime}\right)=0
$$

while the equation of the second one occurs when $u_{k}=u^{S}$ which gives, from (11):

$B C B_{k}^{2}: u^{S}\left(1-\omega^{k-1} \omega^{\prime}\right)-u^{*}-\omega^{k-2}\left[u^{* \prime} *\left(1-\omega^{\prime}\right)+\omega^{\prime} u^{*}(1-\omega)-u^{*}\right]=0$

The equations in (12) and (13) can be put in explicit form with respect several parameters, for example with respect to $\omega^{\prime}$ we get:

$$
\begin{aligned}
B C B_{k}^{1}: \omega^{\prime} & =\frac{u^{*}-u^{S}+\omega^{k-1}\left(u^{* \prime}-u^{*}\right)}{\omega^{k-1}\left(u^{* \prime}-u^{S}\right)} \\
B C B_{k}^{2}: \omega^{\prime} & =\frac{u^{*}-u^{S}+\omega^{k-2}\left(u^{* \prime}-u^{*}\right)}{\omega^{k-2}\left(u^{* \prime}-u^{*}\right)+\omega^{k-1}\left(u^{*}-u^{S}\right)}
\end{aligned}
$$

and these are used to plot the analytical bifurcation curves in Fig.3b, for $k=2, \ldots, 16$, below the region of the 2-cycle, while Fig.3a shows the periodicity regions obtained numerically, where different colors denote different periods.

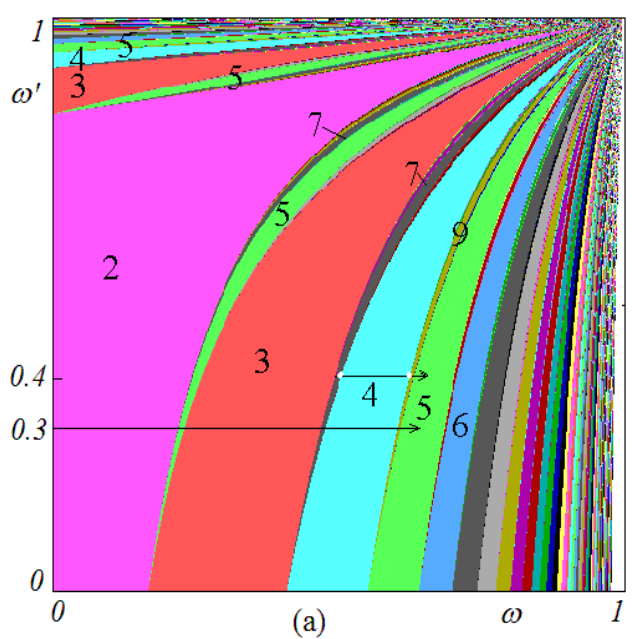

(a)

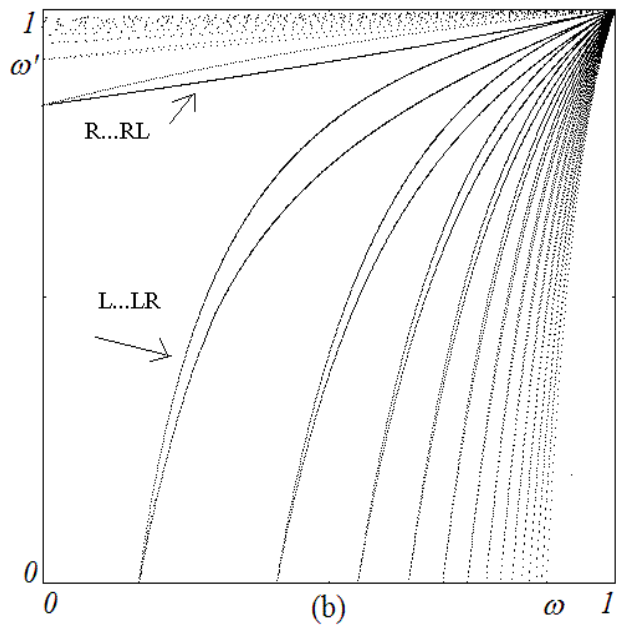

(b)

Fig.3 In (a): two-dimensional bifurcation diagram in the $\left(\omega, \omega^{\prime}\right)$ plane at fixed $u^{*}=0.8, u^{* \prime}=0.2$, and $u^{S}=0.7$. In (b) the bifurcation curves of first degree for $k=2, \ldots, 16$.

Clearly we can also reason differently, searching for maximal $k$-cycles having one point in the region $\mathrm{L}$ and $(k-1)$ points in $\mathrm{R}$ (for any integer 
$k>1$ ), a kind of symmetric case, in which $\mathrm{L}$ and $\mathrm{R}$ are changed into $\mathrm{R}$ and $\mathrm{L}$ respectively. Indeed, by repeating the same reasoning as above, and labelling the $k$-cycle with periodic points $\left\{x_{i}\right\}$ with $x_{1}<u^{S}$ and the other points as $x_{2}>\ldots>x_{k}>u^{S}$, we obtain the same equations as above, in (10), (11), (12) and (13), with the parameters $\omega$ and $u^{*}$ respectively changed in $\omega^{\prime}$ and $u^{* \prime}$ and vice versa, $\omega^{\prime}$ and $u^{* \prime}$ respectively changed in $\omega$ and $u^{*}$. Thus for the analytical expressions of the periodic points we get:

$$
\begin{gathered}
x_{1}=\frac{u^{* \prime}-\omega^{\prime k-1}\left(u^{* \prime}+\omega u^{*}-u^{*}\right)}{\left(1-\omega^{\prime k-1} \omega\right)} \\
x_{k}=\frac{u^{* \prime}+\omega^{\prime k-2}\left[u^{*} *(1-\omega)+\omega u^{* \prime}\left(1-\omega^{\prime}\right)-u^{* \prime}\right]}{\left(1-\omega^{\prime k-1} \omega\right)}
\end{gathered}
$$

The two BCB curves, which we denote by $\overline{B C}_{k}^{i}(i=1,2)$ to distinguish them from the previous ones, are obtained via $x_{1}=u^{S}$ which gives:

$$
\overline{B C B}_{k}^{1}: u^{S}\left(1-\omega^{\prime k-1} \omega\right)-u^{* \prime}+\omega^{\prime k-1}\left(u^{* \prime}+\omega u^{*}-u^{*}\right)=0
$$

and the second one via $x_{k}=u^{S}$ which gives:

$\overline{B C B}_{k}^{2}: u^{S}\left(1-\omega^{\prime k-1} \omega\right)-u^{* \prime}-\omega^{\prime k-2}\left[u^{*} *(1-\omega)+\omega u^{* \prime}\left(1-\omega^{\prime}\right)-u^{* \prime}\right]=0$

These equations are used to plot the analytical bifurcation curves in Fig.3b above the region of the 2-cycle, for $k=3, \ldots, 16$ (as clearly for $k=2$ the two cases give the same BCB curves).

The equations given above are the analytic expressions of the bifurcation curves at which a BCB occurs. For example, the two white points in the periodicity region of the 4-cycle shown in Fig3a (at $u^{*}=0.8$, $u^{* \prime}=0.2, u^{S}=0.7$ and $\left.\omega^{\prime}=0.4\right)$ are used to plot the map in the two cases shown in Fig.2: the 4 -cycle does not exists for $\omega<\omega_{1}=0.51$, at $\omega=\omega_{1}$ the 4 -cycle appears via BCB as shown in Fig.2b (i.e. we have $u_{1}=u^{M}$ and $u_{4}=u^{S}$ ), the 4-cycle exists for parameters inside the periodicity region, $\omega_{1}<\omega<\omega_{2}$, at $\omega=\omega_{2}=0.63$ another BCB occurs, the periodic point $u_{1}$ collides with the boundary of its region (i.e. we have $u_{1}=u^{S}$ ) and the cycle will disappear: it does not exist any longer for $\omega>\omega_{2}$.

Note that the BCB curves given in (12) and (13), as the symmetric ones in (16) and (17), are generic, and hold whichever is the position of the discontinuity point $u^{S}$, and a change in its value gives curves which are only slightly deformed.

Moreover, it is worth noticing that following similar arguments it is possible to find also the boundaries of the other bifurcation curves. In 
fact, besides the periodicity regions of first degree associated with the maximal cycles there are infinitely many (countable) periodicity regions of cycles with different number of periodic points in $L$ and $R$. The simplest well known mechanism to find the periods that characterize these infinite sequences of periodicity regions is that between any two regions having periods $k_{1}$ and $k_{2}$ there exists also a regions having period $k_{1}+k_{2}$ (see the numbers of the periods indicated in Fig.3a: between the regions of period 2 and 3 a region of period 5 exists, and between 5 and 2 we can see 7 , and so on...).

To be more specific, in the description of the periodicity regions we can associate a number to each region, which may be called rotation number, in order to classify all the periods and several cycles with the same period. In this notation a periodic orbit of period $k$ is characterized not only by the period but also by the number of points in the two branches separated by the discontinuity point $u^{S}$ (denoted by $\mathrm{L}$ and $\mathrm{R}$ respectively). We can say that a cycle has a rotation number $\frac{p}{k}$ if a $k$-cycle has $p$ points on the $\mathrm{L}$ side and the others $(k-p)$ on the $\mathrm{R}$ side. Then, between any pair of periodicity regions associated with the rotation numbers $\frac{p_{1}}{k_{1}}$ and $\frac{p_{2}}{k_{2}}$ there exists also the periodicity region associated with the rotation number $\frac{p_{1}}{k_{1}} \oplus \frac{p_{2}}{k_{2}}=\frac{p_{1}+p_{2}}{k_{1}+k_{2}}$ (where $\oplus$ stands for the so-called Farey composition rule, or summation rule, see for example in $[20])$.

Then, following [28] and [36] (see also [37] pp. 56-61 and pp. 80-84), between any pair of consecutive regions of first degree, say with rotation numbers $\frac{1}{k_{1}}$ and $\frac{1}{k_{1}+1}$, we can construct two infinite families of periodicity regions, called regions of second degree via the sequence obtained by adding with the Farey composition rule $\oplus$ iteratively the first one or the second one, i.e. $\frac{1}{k_{1}+1} \oplus \frac{1}{k_{1}}=\frac{2}{2 k_{1}+1}, \frac{2}{2 k_{1}+1} \oplus \frac{1}{k_{1}}=\frac{3}{3 k_{1}+1}, \frac{3}{3 k_{1}+1} \oplus \frac{1}{k_{1}}=$ $\frac{4}{4 k_{1}+1}, \ldots$ and so on, that is:

$$
\frac{n}{n k_{1}+1} \text { for any } n>1
$$

and $\frac{1}{k_{1}} \oplus \frac{1}{k_{1}+1}=\frac{2}{2 k_{1}+1}, \frac{2}{2 k_{1}+1} \oplus \frac{1}{k_{1}+1}=\frac{3}{3 k_{1}+2}, \frac{3}{3 k_{1}+2} \oplus \frac{1}{k_{1}+1}=\frac{4}{4 k_{1}+3} \ldots$, that is:

$$
\frac{n}{n k_{1}+n-1} \text { for any } n>1
$$

which give two sequences of regions accumulating on the boundaries of the two starting ones.

Clearly, this mechanisms can be repeated: between any pair of contiguous "regions of second degree", for example $\frac{n}{n k_{1}+1}$ and $\frac{n+1}{(n+1) k_{1}+1}$, we can construct two infinite families of periodicity regions, called "regions of third degree" via the sequence obtained by adding with the composition rule $\oplus$ iteratively the first one or the second one. And so on. All 
the rational numbers are obtained in this way, giving all the infinitely many periodicity regions.

We also remark (to be used in Section 5) that, by using (7), the product $\lambda=\omega^{k-1} \omega^{\prime}<1$ gives the eigenvalue of the $k$-cycle with symbolic sequence $\mathrm{L} . . . \mathrm{LR}=\mathrm{L}^{k-1} \mathrm{R}$, inside its region of existence, while $\lambda=\omega \omega^{\prime k-1}<1$ gives the eigenvalue of the $k$-cycle associated with symbolic sequence $\mathrm{R} . . . \mathrm{RL}=\mathrm{R}^{k-1} \mathrm{~L}$. This for the maximal cycles. However other periodicity regions exist between two consecutive regions, whose rotation numbers are obtained by using the Farey composition rule $\oplus$ as explained above, and the eigenvalue of a cycle obtained by the composition rule $\oplus$ is the product of the eigenvalues of the two cycles entering in the composition. That is, let us consider for example the cycle with rotation number $\frac{n}{n k_{1}+1}$ (obtained combining the cycle with rotation number $\frac{1}{k_{1}+1}$ and $(n-1)$ times the cycle with rotation $\left.\frac{1}{k_{1}}\right)$, then the eigenvalues of the cycle is

$$
\left(\lambda_{k_{1}}\right)^{(n-1)} \lambda_{k_{1}+1}=\left(\omega^{k_{1}-1} \omega^{\prime}\right)^{(n-1)}\left(\omega^{k_{1}} \omega^{\prime}\right)
$$

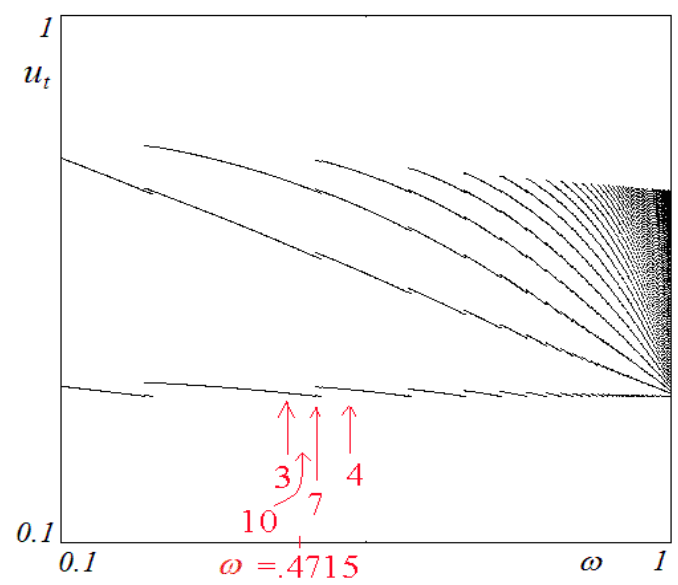

Fig.4 Bifurcation diagram in $\omega$ at fixed $\omega^{\prime}=0.3, u^{*}=0.8, u^{* \prime}=0.2$, and

$$
u^{S}=0.7 \text {. }
$$

It is worth to notice that all the periodicity regions determined in this case of regular dynamics never overlap, and this implies that the coexistence of different $k$-cycles is impossible. This has already been proved in [28], [36], [37]. Moreover, considering the complementary region in the parameter space, not belonging to any periodicity region or to their boundaries, the map has no attracting cycle in $I$, and the dynamics are topologically conjugated to those of a linear rotation (or linear circle map, see [37]) with irrational rotation number. That is, any point in $I$ has a quasiperiodic trajectory which is dense in the whole interval $I$. We remark that no chaos can occur as there is no sensitivity with respect 
to the initial conditions (initial conditions close to each other will have iterated points forever close to each other). Also it is not possible to have a Cantor set as attractor, as a Cantor set requires the existence of infinitely many coexisting unstable cycles, which cannot occur in this regime of stable dynamics.

In Fig. 4 we show the bifurcation diagram which plots the asymptotic behavior of the state variable $u_{t}$ as the slope $\omega$ increases along the line with an arrow shown in Fig.3a (at $\omega^{\prime}=0.3$ fixed). It is worth noticing that the main part of the parameter plane $\left(\omega, \omega^{\prime}\right)$ is covered by the regions associated with the maximal cycles, with symbolic sequence L...LR, and increasing $\omega$ the number of states in the $\mathrm{L}$ region increases (as it increases with the period). Also from Fig.4, which is numerically obtained, it is difficult to see that the existing periods are more than the maximal ones, but some cycles of the second degree are visible: Between the cycles of periods 3 and 4 we see the period 7 , and enlarging we can see also another cycle of the second degree: between the period 3 and 7 there is a cycle of period 10, but its region of existence is so thin that it is almost undetectable in a real application of the model. This enforces the results of the applied model: the good region $\mathrm{L}$ is dominant, and the state R occurs occasionally and once, as it is followed by a sequence of $\mathrm{L}$ states. This will be remarked also in the next example, let us conclude here stating that we have so proved the following:

Theorem 1. Let $\omega$ and $\omega^{\prime}$ be positive and less than 1 in the model (1), and $u^{* \prime}<u^{S}<u^{*}$, then an invariant interval $I=\left[u^{m}, u^{M}\right]$ exists, which is globally absorbing and whose basin of attraction is $\mathcal{B}(I)=$ ] $-\infty,+\infty$ [. Inside I the dynamics are regular, associated with attracting cycles of any period or quasiperiodic trajectories. The regions of existence of the maximal cycles are bounded by $B C B$ curves given in (12), (13), (16) and (17). The set of parameter values not belonging to periodicity regions or their boundaries are associated with quasiperiodic dynamics in $I$.

\section{$3.10<\omega^{\prime}<\omega<1$}

In this subsection we are going to show how the widest regions in the parameter space are those associated with the preferred regime, in which several states are on the $\mathrm{L}$ side and only a few states on the $\mathrm{R}$ one. In Fig.3 we have already shown this in the two-dimensional parameter plane $\left(\omega, \omega^{\prime}\right)$ (and also at different values of the other parameters the figures are qualitatively the same). Now we show that similar results hold also when we vary a different parameter. We illustrate here the particular 
case considered in [16], that is with the restriction

$$
0<\omega^{\prime}<\omega<1
$$

when the parameter $u^{S}$, i.e. the discontinuity point, is let to vary in its feasible region, that is between $u^{* \prime}$ and $u^{*}$. Fig.5 shows the bifurcation curves, and it can be seen that on the right side of the region of the 2cycle we have the periodicity regions associated with the maximal cycles with symbol sequence L...LR, while the others occur moving to the left. This is an important result as indeed, the higher the value of $u^{S}$ (point in which the switching regime occurs) the longer is the stable branch on the $\mathrm{L}$ side, and thus the longer is the sequence of states on the $\mathrm{L}$ regime. This can be better appreciated in a one-dimensional bifurcation diagram, as shown in Fig.6a, which illustrates the dynamic behavior of $u_{t}$ as a function of $u^{S}$, when the parameter moves along the line with an arrow shown in Fig.5a. From values of $u^{S}$ higher than 0.4 the dynamics are mainly in the good regime $\mathrm{L}$, and the $\mathrm{R}$ regime occurs rarely. It is clear that from a mathematical point of view we know the existence of other cycles obtained combining different cycles with the symbolic sequence L...LR, as shown in the enlargement of Fig.6b, but their existence is related to so thin regions which may be considered rare to occur.

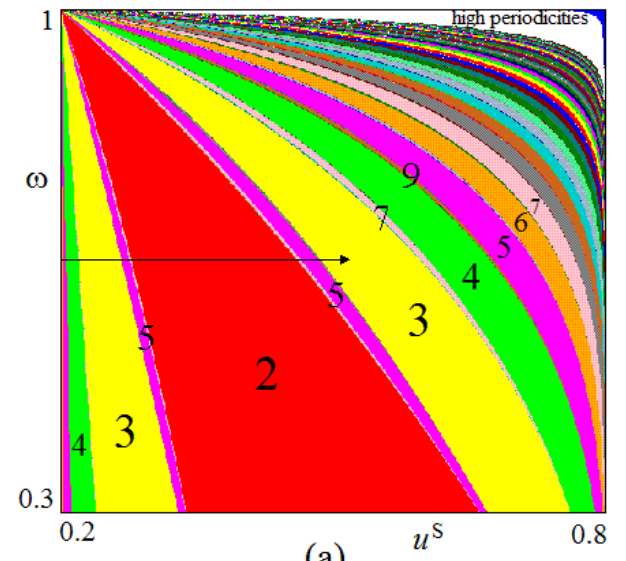

(a)

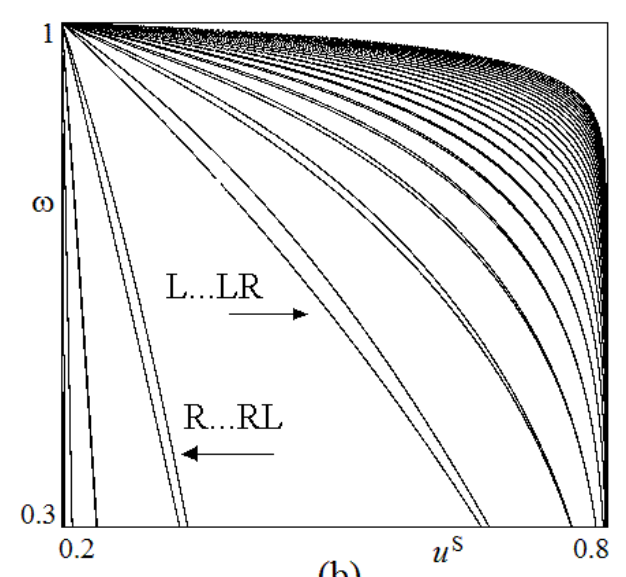

(b)

Fig.5. Periodicity regions of the principal $k$-cycles in the two-dimensional parameter plane $\left(u^{S}, \omega\right)$ at fixed values of the other parameters: $u^{* \prime}=0.2$,

$$
u^{*}=0.8 \text {, and } \omega^{\prime}=0.3 \text {. }
$$

We can see that fixing a value of $u^{S}$ and increasing $\omega$ all the periodicity regions are crossed. 

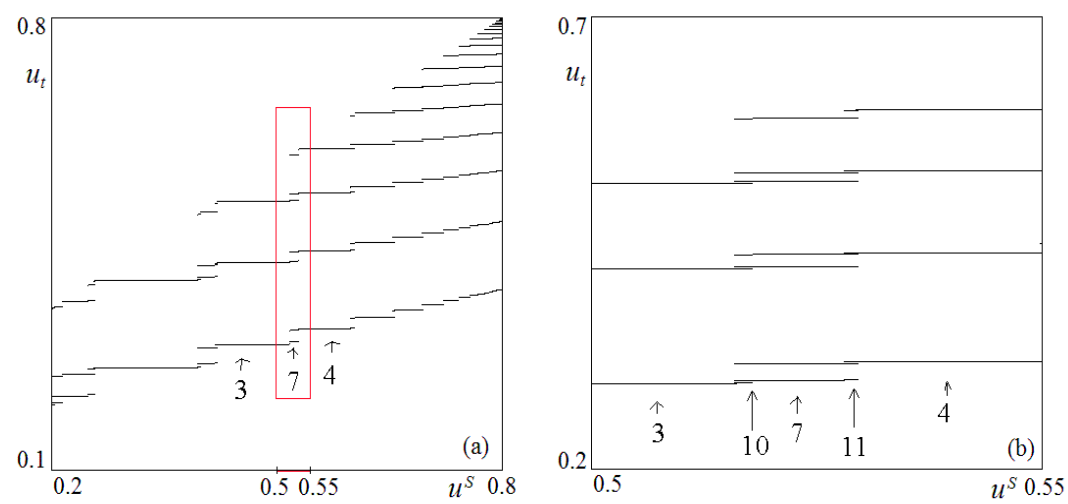

Fig.6 Bifurcation diagram in $u^{S}$ at fixed $\omega=0.75, \omega^{\prime}=0.3, u^{*}=0.8$, $u^{* \prime}=0.2$. Fig. (b) is an enlargement of the portion of the bifurcation diagram (a) evidenced by the red rectangle.

From the one-dimensional bifurcation diagram showing the related dynamics of $u_{t}$ reported in Fig. 6 it can be seen that the qualitative shape of the bifurcation diagram recalls the "devil staircase" ([20]). Indeed, all the cycles of the period-adding scheme described in the previous section exist for parameters in suitable intervals, and a kind of self-similar property occurs, as between any two periods there are infinitely many other related periods. And, as previously remarked, from a mathematical point of view we know that all the bifurcation curves are limit sets of other bifurcation curves.

Clearly similar comments hold also if we consider fixed $u^{S}>0.4$ increasing $\omega$ : we can say that there is a predominance of states belong to the $\mathrm{L}$ region.

\section{Analysis of case (II) $\omega>1, \omega^{\prime}>1$}

In this section we consider the case in which the slopes are both higher then 1 . If some $k$-cycle with $k \geq 1$ exists, then the related eigenvalue is always higher than 1 , and thus it is unstable. It is easy to see that if none or only one fixed point exists then all the dynamics are divergent (except for the fixed point). It follows that bounded dynamics in some intervals can occur only when both the two fixed points exist, although it is a necessary condition, not sufficient. In fact, let us consider the fixed point $P^{*}=u^{*}$ in the $\mathrm{L}$ region having the maximum $u^{M}=(1-\omega) u^{*}+\omega u^{S}$, and the fixed point $Q^{*}=u^{* \prime}$ belonging to the $\mathrm{R}$ region giving the minimum $u^{m}=\left(1-\omega^{\prime}\right) u^{* \prime}+\omega^{\prime} u^{S}$. Then an invariant absorbing interval $I=$ $\left[u^{m}, u^{M}\right]$ exists iff it is inside the two repelling fixed points, that is iff $P^{*} \leq u^{m}$ and $u^{M} \leq Q^{*}$, i.e.:

$$
u^{*} \leq\left(1-\omega^{\prime}\right) u^{* \prime}+\omega^{\prime} u^{S} \text { and }(1-\omega) u^{*}+\omega u^{S} \leq u^{* \prime}
$$


The qualitative shape of the model in this case is shown in Fig.7a. All the points on the right of the fixed point $P^{*}$ and on the left of the fixed point $Q^{*}$ have trajectories entering the absorbing interval $I$, from which the trajectory cannot escape. So that the basin of attraction of $I$ is the interval bounded by the two unstable fixed points:

$$
\mathcal{B}(I)=] P^{*}, Q^{*}[=] u^{*}, u^{* \prime}[
$$

while initial conditions lower than the fixed point $P^{*}$ diverge to $-\infty$, and those starting with values of the unemployment higher than $u^{* \prime}=Q^{*}$ are divergent to $+\infty$.
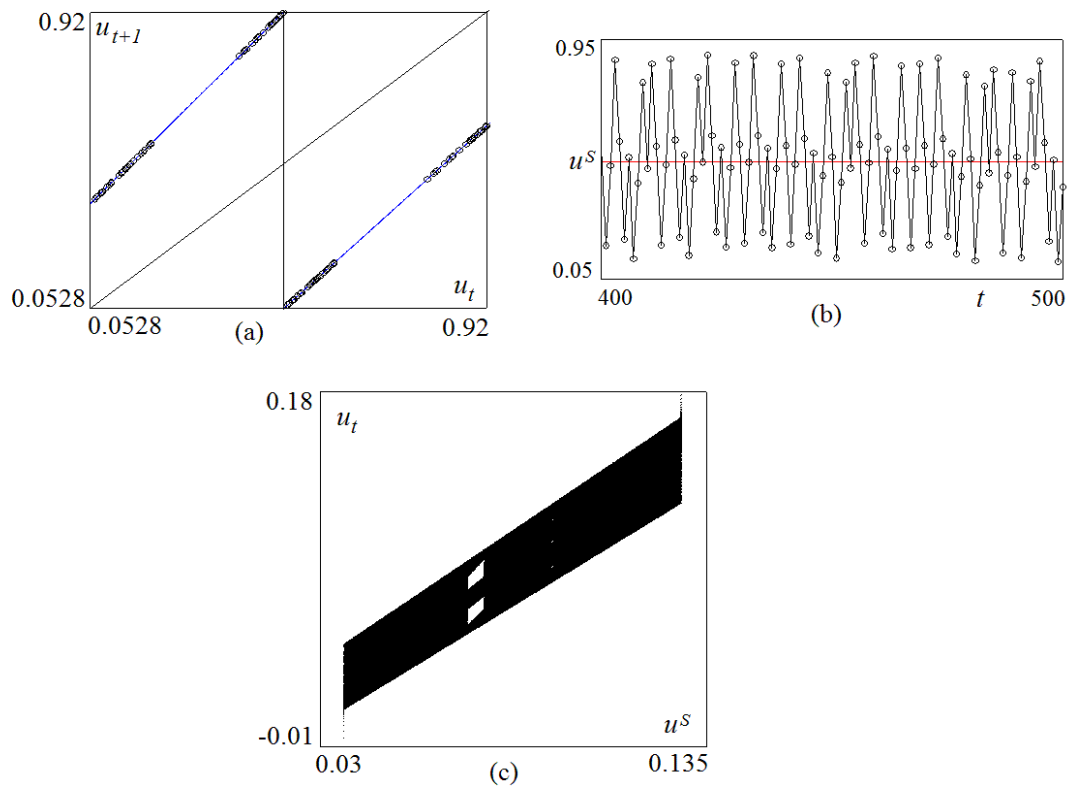

Fig.7 Parameter values $u^{*}=0.01, u^{* \prime}=0.166, \omega=1.324, \omega^{\prime}=1.204$ and $u^{S}=0.07$. Graph of the map in (a), when there are 3 -cyclical chaotic intervals. Versus time trajectory in (b). Bifurcation diagram in (c).

Inside $I$ the attracting set consists in $k$-cyclical $(k \geq 1)$ chaotic intervals, in which the aperiodic trajectories are dense. We notice that it is not true that the dynamics are chaotic in the whole interval $I$, anyhow the $\mathrm{k}$-th iterate of the map has invariant chaotic intervals, and an absolutely continuous invariant measure exists (cfr. [12], [16]). An example of the versus time trajectory in the case with three chaotic intervals shown in Fig.7a is reported in Fig.7b. This is a classical case of pure chaos in cyclical chaotic intervals in the phase space (for the independent variable $u_{t}$ ), and also occurring in an interval of values for each parameter. This is an important property in the applied context, often missed. It is well known that also in smooth models this pure chaos exists (we may think at several cases modelled for example with the logistic map, and cyclical 
chaotic intervals), but often it cannot occur for an interval of parameter values. Differently, in piecewise linear models, when a chaotic regime occurs, then it is persistent as a function of the parameters, and this property is often called of robust chaos. An example is shown in the one-dimensional bifurcation diagram of Fig.7c. From Fig.7c it can be seen that starting from a value of $u^{S}$ at which we have bounded chaotic intervals, then both increasing and decreasing the parameter we end in a bifurcation which is followed by divergent dynamics. Indeed both such bifurcations are contact bifurcations of the invariant absorbing interval. If the invariant interval $I$ has a contact with its basin boundary in the point $P^{*}$, which occurs when $u^{*}=\left(1-\omega^{\prime}\right) u^{* \prime}+\omega^{\prime} u^{S}$, say at $u^{S}=u_{m}^{S}$ where

$$
u_{m}^{S}=\frac{u^{*}-\left(1-\omega^{\prime}\right) u^{* \prime}}{\omega^{\prime}}
$$

then (for $u^{*}>\left(1-\omega^{\prime}\right) u^{* \prime}+\omega^{\prime} u^{S}$ i.e. $u^{S}<u_{m}^{S}$ ) the invariant interval is destroyed, after which almost all the trajectories below $Q^{*}$ are divergent to $-\infty$ (except those of an invariant repelling Cantor set of points of zero Lebesque measure). An example of this case is shown in Fig.8a. The result of the contact bifurcation is thus very dangerous. Its effect is due to the fact that the fixed point $P^{*}$ is on the boundary of the basin $\mathcal{B}(I)$ and also on the boundary of the basin of trajectories divergent to $-\infty$ as $\mathcal{B}(-\infty)=\left(-\infty, P^{*}\right)$. Thus after the contact the invariant interval ends to exist, and the basin $\mathcal{B}(-\infty)$ expands above $P^{*}$.

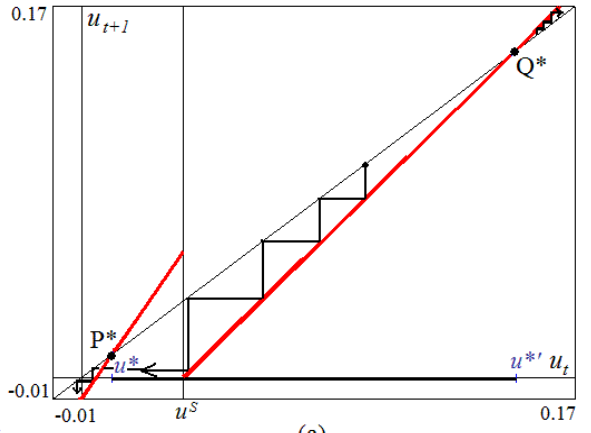

(a)

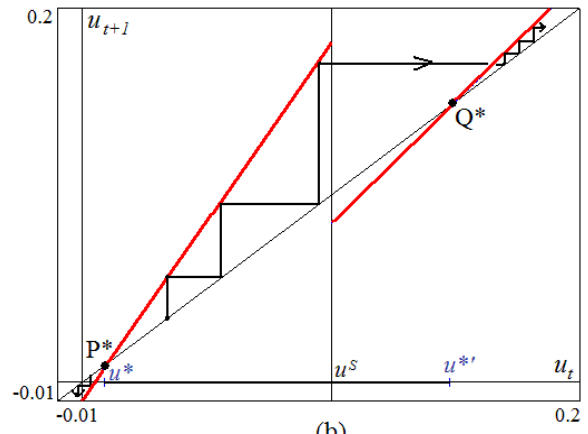

(b)

Fig.8 shape of the map at $u^{*}=0.01, u^{* \prime}=0.15, \omega=1.9, \omega^{\prime}=1.3$ (as in Fig.7) and $u^{S}=0.035$ in (a), $u^{S}=0.1$ in (b).

Similarly, when the invariant interval $I$ has a contact with its basin boundary in the point $Q^{*}$, which occurs when $(1-\omega) u^{*}+\omega u^{S}=u^{* \prime}$, say at $u^{S}=u_{M}^{S}$ where

$$
u_{M}^{S}=\frac{u^{* \prime}-(1-\omega) u^{*}}{\omega}
$$

then (for $u^{* \prime}<(1-\omega) u^{*}+\omega u^{S}$ i.e. $u^{S}>u_{M}^{S}$ ) the invariant interval is destroyed, after which almost all the trajectories of the points above $P^{*}$ are 
divergent to $+\infty$. That is, now the contact bifurcation occurs between the basin $\mathcal{B}(I)$ and the boundary of the basin of trajectories divergent to $+\infty, \mathcal{B}(+\infty)=\left(Q^{*},+\infty\right)$, and after the contact the invariant interval ends to exist, and the basin $\mathcal{B}(+\infty)$ expands below $Q^{*}$. An example of this case is shown in Fig.8b. We have so proved the following:

Theorem 2. Let $\omega>1$ and $\omega^{\prime}>1$ in the model (1), then an invariant absorbing interval $I=\left[u^{m}, u^{M}\right]$ with basin $\left.\mathcal{B}(I)=\right] u^{*}, u^{* \prime}[$ exists iff (19) holds. For $u_{m}^{S}<u^{S}<u_{M}^{S}$ there is robust chaos in with cyclical chaotic intervals in I. When one of the two inequalities in (19) is not verified then almost all the trajectories are divergent.

\section{Analysis of case (III)}

In this section we turn to the dynamics associated with case (III). Let us consider the case with

$$
0<\omega<1, \omega^{\prime}>1
$$

as clearly a similar reasoning can be applied in the case $0<\omega^{\prime}<1$ and $\omega>1$ (exchanging $\omega^{\prime}$ with $\omega$ and vice versa). However, due to a suitable interpretation of our model, it is preferable to have a stable slope in the branch associated with the region $\mathrm{L}$, where it is good to have many states with low unemployment, while in the branch associated with high unemployment it is reasonable to assume a high speed and thus a high slope. Indeed this case may be considered as quite reasonable and interesting to investigate.

We don't consider the case in which $Q^{*}$ does not exist, as this case is not suitable for our interpretation: if $Q^{*}$ does not exist then for any $u_{t}>u^{S}$ the dynamics are divergent to $+\infty$. If $P^{*}$ exists then it attracts all the points $u_{t}<u^{S}$, otherwise everything is divergent.

It follows that when $u^{m}<u^{S}<u^{M}$ then $Q^{*}$ exists (whereas $P^{*}$ does not exist), and an invariant absorbing interval $I=\left[u^{m}, u^{M}\right]$ exists, which attracts all the points at his left, where the increasing function $\theta_{L}$ has a stable slope, and from the right side $I$ attracts all the points below $Q^{*}$. So we have $\mathcal{B}(I)=\left(-\infty, Q^{*}\right)=\left(-\infty, u^{* \prime}\right)$, while trajectories are diverging to $+\infty$ for $u>u^{* \prime}$.

Let us start with the obvious consideration that under such conditions we may expect both regular dynamics (with attracting cycles or quasiperiodic trajectories) and chaotic dynamics. The most striking result is that we cannot have them both simultaneously (as it occurs in the smooth case, for example in the standard logistic map when the parameter is in a periodic window after the Feigenbaum point), and chaos is always robust. The most obvious expectation in the case (III) is a 
kind of progressive destabilization of the possible cycles, while this does not occur. As we shall show, the transition from a regular regime to a chaotic one occurs instantaneously for all the possible cycles. That is to say, before a given condition all the possible cycles are stable, while after all the cycles are unstable. This is clearly a very particular bifurcation. This was already proved in [26] for a discontinuous map $F(x)$ increasing the interval $[0,1]$ into itself, with a discontinuity in $x=d \in] 0,1[$ with jump equal to 1 , for which there are regular (resp. chaotic) dynamics for $F(0)>F(1)$ (resp. $F(0)<F(1)$ ) and the bifurcation occurs at $F(0)=F(1)$. The same result is proved in this section for our map: which has regular (resp. chaotic) dynamics for $u^{*}<u^{* \prime}\left(\operatorname{resp} . u^{*}>u^{* \prime}\right)$ and the bifurcation occurs at $u^{*}=u^{* \prime}$, with a description of the dynamics at the bifurcation value, which are homeomorphic to a linear rotation with rational or irrational rotation number, when the points of the absorbing interval $I=\left[u^{m}, u^{M}\right]$ are periodic or quasiperiodic, respectively.

To illustrate the dynamics of this case (III) let us start with the description of the possible bifurcation diagrams in the one- and twodimensional parameter space. Fig.9a shows the two-dimensional parameter space $\left(u^{*}, \omega^{\prime}\right)$, the slope in the region $\mathrm{L}$ is fixed at $\omega=0.3$ while $u^{S}=0.1$ and $u^{* \prime}=0.15$. In that bifurcation diagram it is shown a stability region of the fixed point $P^{*}\left(=u^{*}\right)$ which undergoes a BCB when the fixed point merges with the discontinuity point, at $u^{*}=u^{S}$ (here at $\left.u^{*}=u^{S}=0.1\right)$. When the fixed point $P^{*}$ exists it is the unique attracting set in $I$. When it disappears by BCB the dynamics enter in a different stability region: no fixed point exists inside the absorbing interval $I$, but stable cycles of several periods or quasiperiodic trajectories. This is shown in the rectangle for $u^{S}<u^{*}<u^{* \prime}$. Inside that region the two BCB curves of the 2-cycle are also drown, by using the equations determined in Section 3. On its right side there are the regions associated with the families R...RL while on its left are those of the families L...LR. For any point in this rectangular region the dynamics are bounded and stable, i.e. for points in a periodicity region a unique attracting cycle exists in $I$, for a set of zero measure of points not belonging to a closed periodicity region the trajectories are quasiperiodic in $I$. The dynamics change from regular to robust chaos at the bifurcation occurring on vertical line in Fig.9a at $u^{*}=u^{* \prime}=0.15$. To prove that this bifurcation is particular we make use of the equations of the BCB curves. 

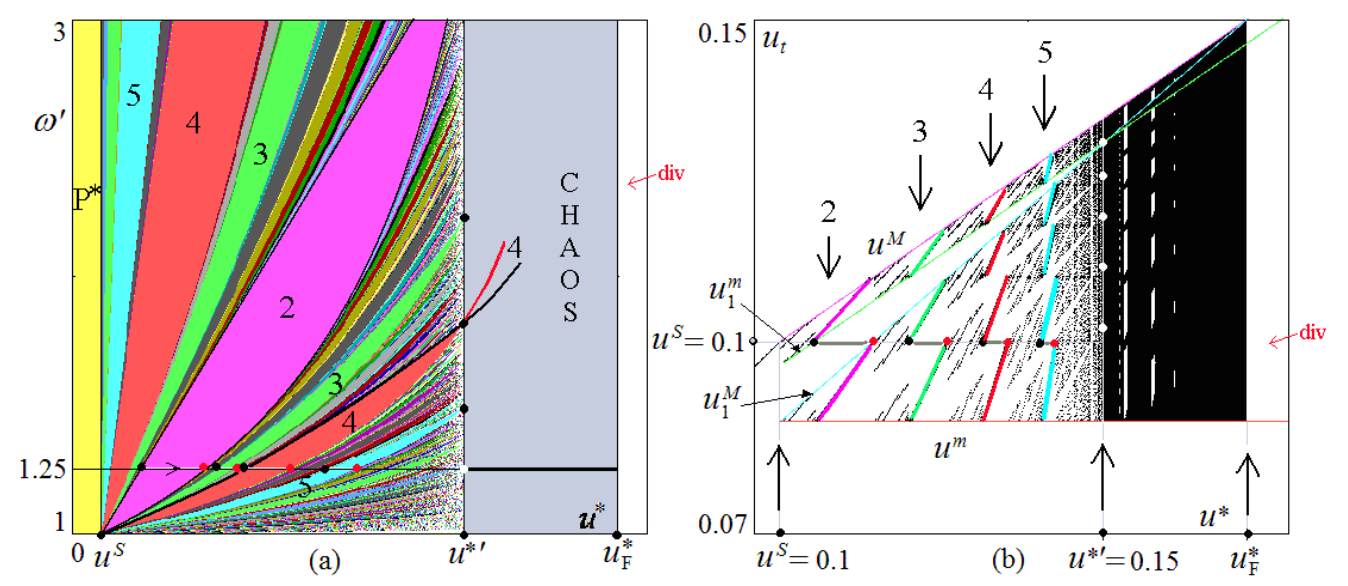

Fig.9 In (a): two-dimensional bifurcation diagram in the $\left(u^{*}, \omega^{\prime}\right)$ plane at fixed $\omega=0.3, u^{* \prime}=0.15$, and $u^{S}=0.1$. In (b) one-dimensional bifurcation diagram showing $u_{t}$ as a function of $u^{*}$ at $\omega^{\prime}=1.25$. The value of $u_{F}^{*}$ is $\simeq 0.1714$.

In Fig.9a it can be seen that the boundaries of the regions associated with the maximal cycles of period 3,4 and 5 intersect exactly at $u^{*}=$ $u^{* \prime}$. In fact, from the analytical expressions of the bifurcation curves given in (12), (13), (16) and (17) we can see (for example by using the explicit expression of $\omega^{\prime}$ as a function of $u^{*}$ ) that all these curves are increasing with $u^{*}$ and are intersecting at $u^{*}=u^{* \prime}$. Then inserting $u^{*}=u^{* \prime}$ in the BCB curves given in (12) and (13) we obtain the equation $\left(u^{S}-u^{*}\right)\left(1-\omega^{k-1} \omega^{\prime}\right)=0$ and having $u^{*} \neq u^{S}$ we get the following condition:

$$
u^{*}=u^{* \prime} \Longrightarrow \omega^{k-1} \omega^{\prime}=1
$$

while inserting $u^{*}=u^{* \prime}$ in (16) and (17) we get (symmetrically):

$$
u^{*}=u^{* \prime} \Longrightarrow \omega \omega^{\prime k-1}=1
$$

Notice that the product $\lambda=\omega^{k-1} \omega^{\prime}$ (which is also a continuous function, increasing with respect both arguments) gives the eigenvalue of the $k$-cycle associated with the BCB curves of type L...LR (as we have remarked in Section 3). It follows that for $u^{*}<u^{* \prime}$ we have $\lambda=\omega^{k-1} \omega^{\prime}<1$ inside the region between each pair of BCB curves, which must intersect at $u^{*}=u^{* \prime}$ where we have the eigenvalue $\lambda=\omega^{k-1} \omega^{\prime}=1$ and for $u^{*}>u^{* \prime}$ the bifurcation curves are crossing and we have the eigenvalue $\lambda=\omega^{k-1} \omega^{\prime}>1$ where the cycle exists. The same reasoning applies for the symmetric family. As an example consider the periodicity region of the 4-cycle in Fig.9a: as the boundaries are increasing functions the curves must intersect at $u^{*}=u^{* \prime}$ and cross at $u^{*}>u^{* \prime}$ (the upper and lower boundaries are exchanged), and the 4-cycle exists for parameter 
points between the two curves. Regarding its eigenvalue $\lambda=\omega \omega^{\prime 3}$ we have that increasing $u^{*}$ for $u^{*}<u^{* \prime} \omega^{\prime}$ is increasing and $\lambda=\omega \omega^{\prime 3}<1$ is also increasing; for $u^{*}=u^{* \prime}$ we have $\lambda=\omega \omega^{\prime 3}=1$ and increasing $u^{*}$ for $u^{*}>u^{* \prime}$ we have $\omega^{\prime}$ increasing and $\lambda=\omega \omega^{\prime 3}>1$ also increasing. Thus the cycle is stable for $u^{*}<u^{* \prime}$ and unstable for $u^{*}>u^{* \prime}$.

Up to now we have considered only the maximal cycles, however the other cycles (see Fig.9a) exist between the BCB curves which are obtained by using the Farey composition rule $\oplus$ as explained in Section 3. Moreover, as already noticed, the eigenvalue of a cycle obtained by the composition rule $\oplus$ is the product of the eigenvalues of the two cycles entering in the composition. Let us consider for example the cycle with rotation number $\frac{n}{n k_{1}+1}$ (obtained combining the cycle with rotation number $\frac{1}{k_{1}+1}$ and $(n-1)$ times the cycle with rotation $\left.\frac{1}{k_{1}}\right)$, then the eigenvalues of the cycle is given in (18), thus we have

$$
u^{*}=u^{* \prime} \Longrightarrow \lambda=\left(\lambda_{k_{1}}\right)^{(n-1)} \lambda_{k_{1}+1}=\left(\omega^{k_{1}-1} \omega^{\prime}\right)^{(n-1)}\left(\omega^{k_{1}} \omega^{\prime}\right)=1
$$

More generally, consider any existing cycle inside its proper region, let $m$ be the number of periodic points in the $\mathrm{L}$ side and $n$ the number of periodic points in the $\mathrm{R}$ side (so that the period is $n+m$ ), then its eigenvalue is $\lambda=\omega^{m} \omega^{\prime n}$, and whichever is the value of the slopes of the model with $0<\omega<1$ and $\omega^{\prime}>1$ then from (23), (24) and from (25) we have:

$$
u^{*}=u^{* \prime} \Longrightarrow \lambda=1
$$

and for $u^{*}>u^{* \prime}$ we have ${ }^{8} \lambda>1$ which implies that any existing cycle is unstable. We can so state the following

Proposition. At the particular bifurcation occurring for $u^{*}=u^{* \prime}$ the map in (1) is topologically conjugated with a linear rotation (or linear circle map) with rational or irrational rotation number. The rotation number is rational in all the points of this set in which two BCB curves are intersecting, and all the points in $I=\left[u^{m}, u^{M}\right]$ are periodic with the same period. Otherwise the rotation number is irrational and the trajectories in I are quasiperiodic and dense in $I$.

The dynamics change completely after this particular bifurcation. In fact for $u^{*}>u^{* \prime}$ we have a chaotic regime, that is all the trajectories inside $I$ enter some cyclical chaotic interval, inside which the dynamic are completely chaotic, in the sense of pure chaos. An absolutely continuous invariant measure exists, and this regime is persistent under perturbations. This is due to the fact that as long as an invariant interval $I$ exists, from which the trajectories cannot escape, we must have that either a

\footnotetext{
${ }^{8}$ due to the fact that the boundaries of the periodicity regions are increasing functions and the eigenvalue also is an increasing function of its arguments
} 
trajectory is periodic, and we know that this occurs only on unstable cycles, or it is aperiodic, but then it must be dense in some intervals, either in I or some cyclical chaotic intervals in I, bounded by the images of the local extrema $u^{m}$ and $u^{M}$.

Let us illustrate this via a one-dimensional bifurcation diagram. In Fig.9b we show the values of the unemployment $u_{t}$ as a function of $u^{*}$ for fixed $\omega^{\prime}=1.25$, the horizontal line in Fig.9a, which crosses regions of the families R...RL. As in Section 3, let us denote the periodic points of a maximal $k$-cycle as $\left\{x_{i}\right\}$ with $x_{1}<u^{S}$ and the other points as $x_{2}>\ldots>x_{k}>u^{S}$. The upper line in Fig.9b denotes the maximum value $u^{M}$ while the lower line denotes the minimum value $u^{m}$. In Fig.9b it is clearly evident the regime of stable cycles existing for $u^{*}<u^{* \prime}$ and it is easy to argue that it is chaotic for $u^{*}>u^{* \prime}$ (as we have proved above). In the regular regime we have emphasized the intervals associated with the periodic orbits of period 2, 3, 4 and 5. From the bifurcation diagram it can be seen that the interval of the 2-cycle starts with a BCB in which the periodic points satisfy $x_{2}=u^{S}$ and $x_{1}=u^{m}$ while it closes at a BCB in which $x_{1}=u^{S}$ and $x_{2}=u^{M}$. The interval of the $3-$ cycle starts with a BCB in which the periodic points satisfy $x_{3}=u^{S}$ and it closes at a BCB in which $x_{1}=u^{S}$. The 4 -cycle starts when $x_{4}=u^{S}$ and it closes when $x_{1}=u^{S}$, and similarly for the 5-cycle. Infinitely many stable cycles exist for $u^{*}<u^{* \prime}$, never coexisting.

Also evident are the other lines in that stability region, which bound the asymptotic points: they are the iterates of the points of maximum $u^{M}$ and of minimum $u^{m}$, and it is clearly evident that such lines are intersecting at the $\mathrm{BCB}$ occurring for $u^{*}=u^{* \prime}$ when all the periodic points merge with the discontinuity point. The first iterate of $u^{M}$ belongs to the line of equation

$$
u_{1}^{M}\left(u^{*}\right)=u^{*}(1-\omega) \omega^{\prime}+\left(1-\omega^{\prime}\right) u^{* \prime}+\omega^{\prime} \omega u^{S}
$$

while the first iterate of $u^{m}$ belongs to the line of equation

$$
u_{1}^{m}\left(u^{*}\right)=u^{*}(1-\omega)+\omega\left(1-\omega^{\prime}\right) u^{* \prime}+\omega^{\prime} \omega u^{S}
$$

and these lines intersect at the bifurcation occurring at $u^{*}=u^{* \prime}$, giving $u_{1}^{M}=u_{1}^{m}=u^{*}\left(1-\omega \omega^{\prime}\right)+\omega^{\prime} \omega u^{S}$.

From Fig.9b we also see that there is a final bifurcation curve at a value $u_{F}^{*}$ after which all the dynamics are divergent. This is the contact bifurcation already described in the previous section. That is, a contact bifurcation of the invariant interval $I$ (and thus of its basin of attraction) with the unstable fixed point $Q^{*}$, which occurs when $u^{M}=Q^{*}$, i.e. when $u^{* \prime}=(1-\omega) u^{*}+\omega u^{S}$. Stated in other words, bounded dynamics exists 
iff $u^{*} \leq u_{F}^{*}$ where

$$
u_{F}^{*}=\frac{u^{* \prime}-\omega u^{S}}{(1-\omega)}
$$

and everything is divergent for $u^{*}>u_{F}^{*}$ except for the points of an invariant Cantor set of zero Lebesque measure (which includes all the existing unstable cycles).

We have so proved the following

Theorem 3. Let $0<\omega<1, \omega^{\prime}>1$ and $u^{m}<u^{S}<u^{M}$ in the model (1), then an invariant absorbing interval $I=\left[u^{m}, u^{M}\right]$ with basin $\mathcal{B}(I)=]-\infty, u^{* \prime}\left[\right.$ exists iff (19) $u^{*} \leq u_{F}^{*}$ holds. For $u^{*} \leq u^{S}$ the fixed point $P^{*}$ is the unique attractor. For $u^{S}<u^{*}<u^{* \prime}$ there are regular dynamics (periodic or quasiperiodic), each closed periodicity region is associated with a unique attracting $k$-cycle in $I$. For $u^{* \prime}<u^{*}<u_{F}^{*}$ there is robust chaos, in $k$-cyclical intervals belonging to I for $k \geq 1$. For $u^{*}>u_{F}^{*}$ all the trajectories are divergent, except for an invariant Cantor set of zero Lebesque measure.

\section{Concluding remarks}

The present paper has focused the attention on the dynamics of unemployment and inflation in a model based upon the presence of a NAIRU, the non accelerating-inflation rate of unemployment. In the literature there is a considerable debate ranging from its very existence to its empirical value in the various economic systems. In the present work, two innovations have been brought about. On the one hand, the Phillips curve have been considered in a system perspective and therefore also aggregate demand has been appended. On the other, a regime switching device has been taken into account.

The results of the analysis are worth stressing. First of all, the model allows to consider in a unified manner different phenomena such as inflation and deflation as well as situations of low unemployment and periods of high unemployment. In the second place, the model does not constrain the dynamics to ignore phenomena such as accelerations or decelerations that are typical in periods of turbulence. Finally, the system does not necessarily create explosive situations. This implies that the NAIRU is not necessarily a single value, nor is it reachable and therefore it becomes very difficult to measure. What happens is that an interval of values for unemployment (also stressed by Solow in [48]) is generated, compatible with a bounded situation. From an economic policy perspective, this situation implies that the razor's edge property of the NAIRU can be avoided and more degrees of freedom are allowed.

The dynamics depend very much on the value of the parameters and 
the structure of the model. In particular, three situations have been envisaged, showing in each case the fundamental bifurcations occurring. As the model with regime switching is represented by a discontinuous piecewise-linear map, the dynamic changes are mainly due to border collision bifurcations and contact bifurcations. We have shown that the abrupt change from a regular regime to a chaotic one occurs in case (III) and leading to robust chaos. The same robust chaos also exists in case (II), while the regular region also include case (I). The dynamics in the regular regime have been fully explained, and we have given the analytical equations of the BCB curves of the maximal cycles.

To close this work, some generalization of the model should be considered. First of all, one can increase the dimension of the system in order to make more explicit the relationship between the rate of growth of income and unemployment on one hand, and the credit conditions and money supply on the other. In particular, regime switching can be applied more explicitly to policy regimes. In the second place, one does not need to operate with backward oriented variables. Assuming naive expectations might be reasonable in an environment where the inflation process is a near-unit root process. However, using a fixed expectation scheme regardless of macroeconomic conditions, is difficult to defend. A theory of expectations that takes into account the possibility of evolving forecasting rule, as those suggested by some learning mechanism, should be considered.

Finally, the empirical performance of the model can be improved. Even tough the values of the parameters, as well their changes, remain within the region established by the econometric research, it is true that the model is more capable to frame alternative scenarios than to carry out precise forecasts. Furthermore, the time series properties generated by the model (such as the different time the system spends in different regimes or the asymmetries between expansion and deceleration) can be better investigated and also the structure of the model itself can be improved.

\section{Acknowledgements.}

We wish to thank three anonymous referees and C. Hommes for stimulating suggestions which helped us to significantly improve our work. A financial contribution from the University of Bergamo is kindly acknowledged.

\section{References}

[1] P.Aghion, P. Howitt, Endogenous Growth Theory, The MIT Press, Cambridge, MA, 1998.

[2] G.A. Akerlof, W.T. Dickens, W.T. Perry, Near-rational wage and 
price setting and the long-run Phillips curve, Brookings Papers on Economic Activity 1 (2000), 1-60.

[3] V. Avrutin, M. Schanz, Multi-parametric bifurcations in a scalar piecewise-linear map, Nonlinearity 19 (2006) 531-552.

[4] V. Avrutin, M. Schanz, S. Banerjee, Multi-parametric bifurcations in a piecewise-linear discontinuous map, Nonlinearity 19 (2006) 1875-1906.

[5] L. Ball, N.G. Mankiw, the NAIRU in theory and practice, J. Econ. Perspect. 16 (2002) 115-136.

[6] S. Banerjee, M.S. Karthik, G. Yuan, J.A. Yorke, Bifurcations in One-Dimensional Piecewise Smooth Maps - Theory and Applications in Switching Circuits, IEEE Trans. Circuits Syst.-I: Fund. Theory Appl. 47 (3) (2000) 389-394.

[7] V. Böhm and L. Kaas, Differential savings, factor shares and endogenous growth cycles, J. Econ. Dynam. Control 24 (2000) 965980.

[8] C. Borio, A. Filardo, Globalization and inflation: new cross-country evidence on the global determinants of domestic inflation. (2007) unpublished paper.

[9] L. Corrado, S. Holly, Nonlinear Phillips curves, mixing feedback rules and the distribution of inflation and output, J. Econ. Dynam. Control 28 (2003) 467-492.

[10] R. Day, Irregular growth cycles, Amer. Econ. Rev. 72 (3) (1982) 406-414.

[11] R. Day, W. Shafer, Ergodic fluctuationsin deterministic economic models, J. Econ. Behav. Organ. 8 (1987) 339-361.

[12] R. Day, G. Pianigiani, Statistical dynamics and economics, J. Econ. Behav. Organ. 16 (1991) 37-83.

[13] R. Day, Complex Economic Dynamics, MIT Press, Cambridge, 1994.

[14] M. Di Bernardo, M.I. Feigen, S.J. Hogan, M.E. Homer, Local analysis of C-bifurcations in n-dimensional piecewise smooth dynamical systems, Chaos Solitons Fractals 10 (11) (1999) 1881-1908.

[15] S. Fazzari, P. Ferri, E. Greenbeg, Cash flow, investment and KeynesMinsky cycles, J. Econ. Behav. Organ. 65 (3-4) (2008) 555-572.

[16] P. Ferri, E. Greenberg, R. Day, The Phillips curve, regime switching and the NAIRU, J. Econ. Behav. Organ. 46 (1) (2001) 23-37.

[17] P. Ferri, The labor market and technical change in endogenous cycles, Metroeconomica 58 (4) (2007) 609-33

[18] P. Ferri, The economics of nonlinear cycles, in Scazzieri, R., Sen, A.and Zamagni, S., (eds) Markets, Money and Capital Hicksian, 2008 
[19] L. Gardini, I.Sushko, A. Naimzada, Growing Through Chaotic Intervals, J. Econ. Theory 143 (1) (2008) 541-557.

[20] Hao Bai-Lin, Elementary Symbolic Dynamics and Chaos in Dissipative Systems. World Scientific, Singapore, 1989.

[21] J.R. Hicks, A Contribution to the Theory of Trade Cycle, OxfrodUniversity Press, Oxford, 1950.

[22] C.H. Hommes, H. Nusse, Period three to period two bifurcations for piecewise linear models, J. Econ. (Z. Nationalökon.) 54 (2) (1991) 157-169.

[23] C.H.Hommes, Chaotic dynamics in economic models, Ph.D. Thesis, Wolters-Noodhoff, Groningen, 1991.

[24] C.H. Hommes, E. Nusse, A. Simonovits, Cycles and chaos in a socialist economy, J. Econ. Dynam. Control 19 (1995) (1-2) 155-179.

[25] C.H. Hommes, A reconsideration of Hick's non-linear trade cycle model, Struct. Change Econ. Dynam. 6 (1995) 435-459.

[26] J.P. Keener, Chaotic behavior in piecewise continuous difference equations, Trans. Amer. Math. Soc. 261(2) (1980) 589-604.

[27] R. Layard, S. Nickell, R. Jackman, Unemployment, Oxfrod University Press, Oxford, 1991.

[28] N.N. Leonov, Map of the line onto itself, Radiofisica 3 (3) (1959) 942-956.

[29] N.N. Leonov, Discontinuous map of the straight line, Dohk. Ahad. Nauk. SSSR. 143 (5) (1962) 1038-1041.

[30] Y.L. Maistrenko, V.L. Maistrenko, L.O. Chua, Cycles of chaotic intervals in a time-delayed Chua's circuit, Internat. J. Bifur. Chaos 3 (6) (1993) 1557-1572.

[31] Y.L. Maistrenko, V.L. Maistrenko, S.I. Vikul, L.O. Chua, Bifurcations of attracting cycles from time-delayed Chua's circuit, Internat. J. Bifur. Chaos 5 (3) (1995) 653-671.

[32] Y.L. Maistrenko, V.L. Maistrenko, S.I. Vikul, On period-adding sequences of attracting cycles in piecewise linear maps, Chaos Solitons Fractals 9 (1) (1998) 67-75.

[33] K. Matsuyama, Growing through cycles, Econometrica 67 (2) (1999), 335-347.

[34] C.J. Metcaf, The dynamics of the Stiglitz policy in the RSS model, Chaos Solitons Fractals 37 (2008) 652-661.

[35] H.P. Minsky, Can it happen again,Sharpe, New York, 1982.

[36] C. Mira, Sur les structure des bifurcations des diffeomorphisme du cercle, C.R.Acad. Sc. Paris 287 Series A (1978) 883-886.

[37] C. Mira, Chaotic dynamics, World Scientific, Singapore, 1987.

[38] C. Mira, L. Gardini, A. Barugola, J.C. Cathala, Chaotic Dynamics in two-dimensional noninvertible maps, World Scientific, Singapore, 
1996.

[39] T. Mitra, A sufficient condition for topological chaos with an application to a model of endogenous growth, J. Econ. Theory 96 (1-2) (2001) 133-152.

[40] A. Mukherji, Robust cyclical growth, Internat. J. Econ. Theory 1 (2005) 233-246.

[41] H.E. Nusse, J.A. Yorke, Border-collision bifurcations including period two to period three for piecewise smooth systems, Physica D 57 (1992) 39-57.

[42] H.E. Nusse, J.A. Yorke, Border-collision bifurcations for piecewise smooth one-dimensional maps, Internat. J. Bifur. Chaos 5 (1) (1995) 189-207.

[43] T. Puu, L. Gardini, I. Sushko, Cournot duopoly with kinked linear demand according to Palander and Wald, in T. Puu and I. Sushko (Eds.) Oligopoly and Complex Dynamics: Tools and Models 111146, Springer-Verlag, N.Y. 2002.

[44] T. Puu, L. Gardini, I. Sushko, A Hicksian Multiplier-Accelerator Model with Floor Determined by Capital Stock, J. Econ. Behav. Organ. 56 (3) (2005) 331-348.

[45] T. Puu, I. Sushko, Oligopoly Dynamics, Models and Tools, Springer Verlag, N.Y. 2002.

[46] T. Puu, I. Sushko, Business Cycle Dynamics, Models and Tools, Springer Verlag, N.Y. 2006.

[47] A.M. Sbordone, Globalization and inflation dynamics: the impact of increased competition, NBER Working Paper 13556 (2007) Cambridge (MA).

[48] R.M. Solow, J.B. Taylor, Inflation, Unemployment and Monetary Policy, The MIT Press, Cambridge, MA, 1998.

[49] I. Sushko, T. Puu, L. Gardini, The Hicksian floor-roof model for two regions linked by interregional trade, Chaos Solitons Fractals 18 (2003) 593-612.

[50] I. Sushko, A. Agliari, L. Gardini, Bistability and border-collision bifurcations for a family of unimodal piecewise smooth maps, Discrete and Continuous Dynamical Systems, Serie B 5 (3) (2005) 881-897.

[51] I. Sushko, A. Agliari, L. Gardini, Bifurcation Structure of Parameter Plane for a Family of Unimodal Piecewise Smooth Maps: Border-Collision Bifurcation Curves. Chaos Solitons Fractals 29 (3) (2006) 756-770.

[52] F. Tramontana, L. Gardini, T. Puu, Duopoly games with alternative technologies, J. Econ. Dynam. Control 33 (1) (2009) 250-265.

[53] M. Woodford, Interest and Prices, Princeton University Press, Princeton, 2003. 